\title{
Energy loss of a high-charge bunched electron beam in plasma: Analysis
}

\author{
N. Barov \\ Department of Physics, Northern Illinois University, DeKalb, Illinois 60115, USA
}

J. B. Rosenzweig, M. C. Thompson, and R. B. Yoder

Department of Physics and Astronomy, UCLA, 405 Hilgard Avenue, Los Angeles, California 90095-1547, USA

(Received 13 March 2003; revised manuscript received 29 April 2004; published 10 June 2004)

\begin{abstract}
There has been much recent experimental and theoretical interest in the blowout regime of plasma wakefield acceleration, which features ultrahigh accelerating fields, linear transverse focusing forces, and nonlinear plasma motion. A quantitative understanding of the blowout regime including all these effects has, to this point, been available only through detailed simulations. This paper represents an initial step towards an analytical theory of this regime, in which the mechanism of energy loss in the drive beam is investigated. We find, first from examination of electromagnetic particle-in-cell simulations, and then through analytical investigations, that under short pulse, high-charge conditions, the plasma electrons receive a strong initial push along the direction of beam motion. This nonlinear effect is unanticipated by linear theory, where the return current motion is in the opposite direction. In the limit of short pulses (the $\delta$-function limit), the beam energy loss is shown to be linear in charge even with a nonlinear plasma response dominated by relativistic, electromagnetic effects, despite the fact that the initial plasma electron response changes qualitatively from the familiar electrostatic, nonrelativistic limit.
\end{abstract}

DOI: 10.1103/PhysRevSTAB.7.061301

\section{INTRODUCTION}

The transfer of energy from short, intense electron beams to collective electron plasma oscillations is a critical component of the advanced, high-gradient acceleration scheme known as the plasma wakefield accelerator (PWFA) [1-4]. While the original proposal for the PWFA and related concepts was in the linear regime [1,2], where the plasma oscillations can be considered small perturbations about an equilibrium, nonlinear regimes have been shown to have favorable attributes [3]. For example, in the highly nonlinear "blowout" regime [4], the plasma electrons are ejected from the channel of the intense driving electron beam, resulting in an electron-rarefied region with excellent quality transverse and longitudinal fields. As the longitudinal electromagnetic fields in the rarefied channel are uniform in radius $r$, and the electrostatic focusing fields are linear in $r$, it is possible to stably accelerate a trailing electron bunch in this region. While many aspects of the beam dynamics in this regime are therefore analytically tractable, the plasma dynamics are not. Because of the nonlinearity of the plasma response, and the lack of useful analytical approaches, quantitative predictions concerning plasma behavior in the blowout regime have been deduced from numerical simulations.

In order to motivate the discussion of highly nonlinear plasma excitation, we wish to define a single parameter that expresses the degree of nonlinearity of the plasma dynamics. In doing so, we first state some assumptions about the volume occupied by the drive beam. In order to efficiently excite a wakefield in both linear and nonlinear regimes, the bunch length $\sigma_{z}$ should be smaller than $2 k_{p}^{-1}$
PACS numbers: 52.40.Mj, 29.17.+w, 29.27.-a, 52.75.Di

( $k_{p}=\sqrt{2 \pi r_{e} n_{0}}$, and $n_{0}$ is the unperturbed plasma electron density). In addition, the bunch radius should obey $\sigma_{r} \ll 1[1,4]$, a condition that is interpreted in linear theory as indicating that the beam-induced electric field, and thus the plasma electron response is predominately in the radial direction. The beam can then be assumed to occupy a volume smaller than approximately $k_{p}^{-3}$. We define the dimensionless beam charge as

$$
\tilde{Q}=\frac{N_{b} k_{p}^{3}}{n_{0}}=4 \pi r_{e} k_{p} N_{b}
$$

where $N_{b}$ is the number of beam electrons, and it has been normalized to the number of plasma electrons in volume of a cubic plasma skin depth $k_{p}^{-3}$. Thus, a beam with density $n_{b}=n_{0}$ uniformly distributed within this volume will have $\tilde{Q}=1$. Coupled with our assumption that the beam volume is smaller than $k_{p}^{-3}$, the condition for blowout $\left(n_{b} / n_{0}>1\right)$ will be surely met when $\tilde{Q}>1$, and a highly nonlinear response - blowout - will result under conditions of $\tilde{Q} \gg 1$. The large density perturbation in the blowout regime is accompanied by both relativistic plasma electron motion and strong beam magnetic field effects. The parameter $\tilde{Q}$ thus measures the importance of such effects; when it exceeds unity, they will be strong.

Guided by the conceptions of linear theory, previous studies of the blowout regime have assumed that, in the region of the beam, the plasma electron motion is mostly radial, with a component of the motion counter to the beam direction that arises from the plasma-derived longitudinal deceleration fields. As $\tilde{Q}$ is raised above unity, however, the electromagnetic fields of the beam become 
stronger, and the initially strong outward radial motion of the plasma electrons is increasingly converted to forward longitudinal momentum by the beam's magnetic fields. This forward pushing overcomes the induced decelerating field and the initial longitudinal motion is forward, in contrast to that foreseen by the predictions, valid for $\tilde{Q} \ll 1$, of linear theory. We will show particle-in-cell (PIC) simulations and analytical results in the context of highly nonlinear, $\tilde{Q} \gg 1$, short bunch scenarios where this longitudinal motion can be relativistic, and the forward momentum component can exceed the radial one. The analysis presented below will address how this motion affects the plasma electron density and currents, as well as how it affects the formation of the plasma electromagnetic response.

The development of a full nonlinear theory of the PWFA in the blowout regime is clearly not feasible. We are thus driven to study an analytically tractable limitthat of the infinitesimally short beam-of the relevant physical scenario, which illuminates new, surprising, physical aspects of the beam-plasma interaction. The analytical results deduced in this paper are exact in the limit of an ultrarelativistic $(v \rightarrow c)$, infinitesimally short (a longitudinal $\delta$-function) beam. Unlike linear theory, however, one may not use the $\delta$-function solution as the basis for understanding finite bunch length effects. A more complete simulation study, that both supports and illuminates the results given in this paper, and further extends the analysis of energy loss and gain in the nonlinear PWFA to finite-length beams, is presented in an accompanying paper [5]. Also, the effect on the analysis of finite, but very short $\left(k_{p} \sigma_{z} \ll 1\right)$ beams is discussed in an appendix.

Despite the lack of analytical models for the nonlinear plasma response, it has been noted in a number of studies [6-9] that the beam energy loss rate in the PWFA blowout regime scales as $\sigma_{z}^{-2}$ when the plasma density is adjusted in order to keep $k_{p} \sigma_{z}$ constant. This prediction has led to a number of experiments that employ bunch compressors in order to decrease the electron bunch length $\sigma_{z}$, thus dramatically increasing the transfer of beam energy to the plasma. In recent measurements with compressed beam at FNAL [10], the trailing portion of a $5 \mathrm{nC}$, $14 \mathrm{MeV}, \sigma_{z}=1.2 \mathrm{~mm}$, beam pulse was nearly stopped in $8 \mathrm{~cm}$ of $n_{0} \simeq 10^{14} \mathrm{~cm}^{-3}$ plasma, a deceleration rate of over $150 \mathrm{MeV} / \mathrm{m}$. The large collective field observed in this as well as other recent PWFA experiments $[10,11]$ has led to a proposal to further exploit frequency scaling by using ultrashort, high-charge beams to drive a PWFA in the tens of $\mathrm{GeV} / \mathrm{m}$ range (in the SLAC E-164 experiment), as part of the so-termed "afterburner" concept $[9,12,13]$.

As we shall see below, in the limit of an infinitesimally short beam, when either the plasma density or the beam charge is increased (to increase $\tilde{Q}$ ), the response of the plasma to the beam eventually becomes nonlinear.
Nevertheless, the energy loss rate, surprisingly, still scales linearly with the charge in this limit, even for nonlinear plasma motion. This paper is primarily intended to address the new aspects of the underlying physics of the very high-charge beam-plasma interaction that can be uncovered in the $\delta$-function beam limit. It is further intended to explore how these aspects may contribute to the linearlike wakefield scaling of relativistic beam energy loss in plasma. This discussion of the plasma electrodynamic response produces comparisons with both the linear regime of the PWFA, as well as with the nonlinear regime of the laser-plasma interaction.

\section{PARTICLE-IN-CELL SIMULATIONS OF AN ULTRASHORT ELECTRON BEAM IN PLASMA}

In order to examine the major nonlinear effects that will arise from our analysis of the $\delta$-function beam in plasma, we have performed fully relativistic, axisymmetric electromagnetic PIC simulations using the $2 \mathrm{D}$ code OOPIC [13]. This uniformly populated, very high-charge $(\tilde{Q}=200)$ bunch is taken to have bounding radial dimension $r=a$ such that $k_{p} a \equiv \tilde{a}=0.2$, and full length $L$ such that $k_{p} L=\tilde{L}=0.1$. The most relevant plasma quantities which can be extracted from the PIC results are the density $n$, and longitudinal current density $J_{z}$. These are shown in Figs. 1 and 2, respectively. It can be seen from Fig. 1 that behind the uniform density beam, which is localized in the region near $z=0.0186$ inside of $r \leq$ $0.0005 \mathrm{~m}(\tilde{L}=0.1, \tilde{a}=0.2)$, the region near the beam axis is completely rarefied of electrons. This is expected in such a strong blowout regime scenario.

On the other hand, in the region directly in the vicinity of the beam's longitudinal position, and extending over a fairly wide radial interval outside of the beam, the plasma electron density increases by nearly an order of magnitude. This effect is completely unanticipated by predictions obtained by linear theory $[2,14,15]$, which indicate that the plasma always becomes initially less dense in response to the introduction of the beam. The diminishing of the density in the linear model is attributed to outward radial currents and to longitudinal currents running opposite to the beam current $[15,16]$.

The PIC simulation results of Fig. 2 show that the longitudinal current density $J_{z}$ in this highly nonlinear case also contrasts with the expectations of linear theory. Again, in the direct vicinity of the beam, where linear theory predicts the induced $J_{z}$ to be counter to the beam, it is observed in the simulations to be in the same direction of the beam (as indicated by the red color, the same as the beam current near axis), and extremely large - as large as $e n_{0} c$. We shall see below that the anomalous current response in the nonlinear regime is due to the initial forward velocity of the plasma electrons; this forward velocity is a straightforward consequence of the combined effects of the beam's large magnetic field. 


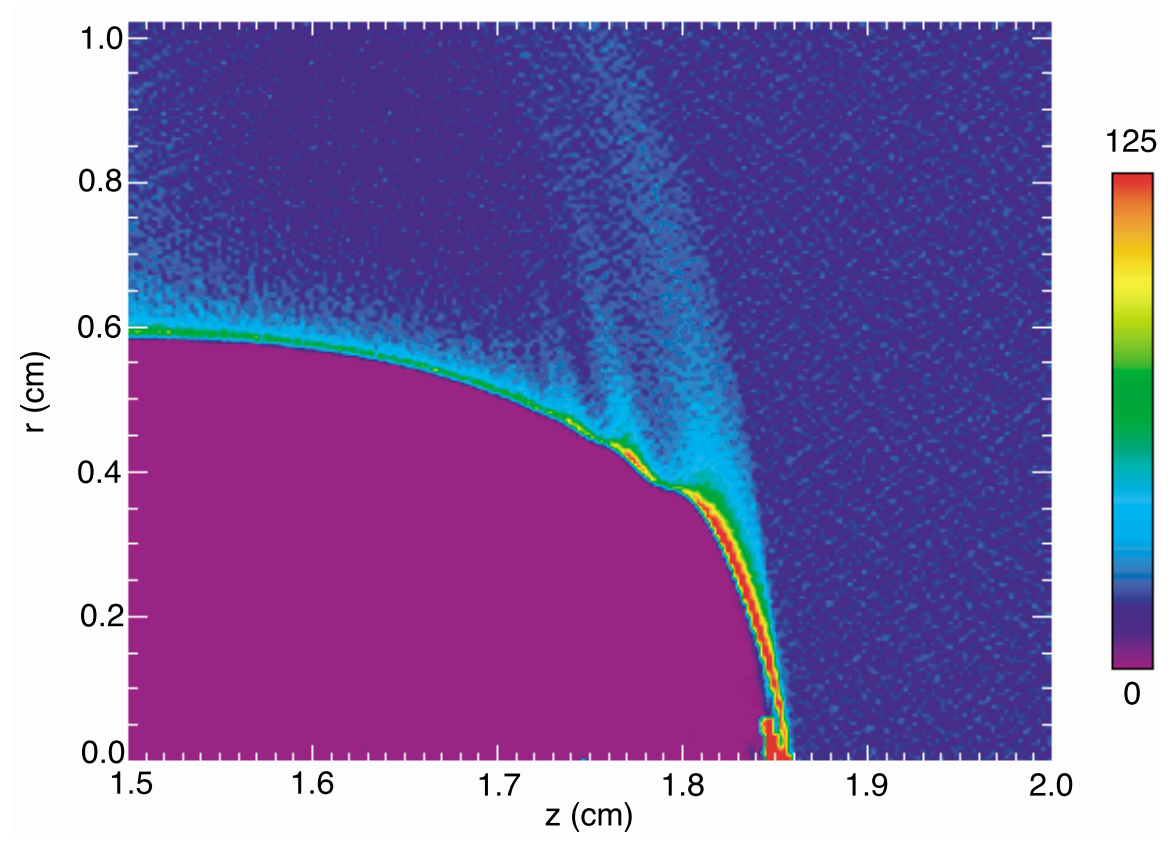

FIG. 1. (Color) Color intensity map of the plasma and beam electron density as a function of $r$ and $z$, with $n_{0}=10^{14} \mathrm{~cm}^{-3}$ indicated as blue (level 15 on the color scale). In this case, the uniform density drive beam is localized near $z=0.0186 \mathrm{~m}$, has a length $\tilde{L}=0.1$, radial extent $\tilde{a}=0.2$, and normalized charge $\tilde{Q}=200$. Note a large density increase in the beam region, followed by a rapid blowout after the beam passes.

Further, a forward velocity component produces, through the constraints of the continuity relation, an enhancement of the plasma electron density, yielding in the end the large observed longitudinal current densities that have the same sign as those of the beam.

\section{NONLINEAR PLASMA EXCITATION BY A RELATIVISTIC ELECTRON BEAM}

As stated above, the general case of a high-charge bunch, with relativistic plasma electrons and large plasma

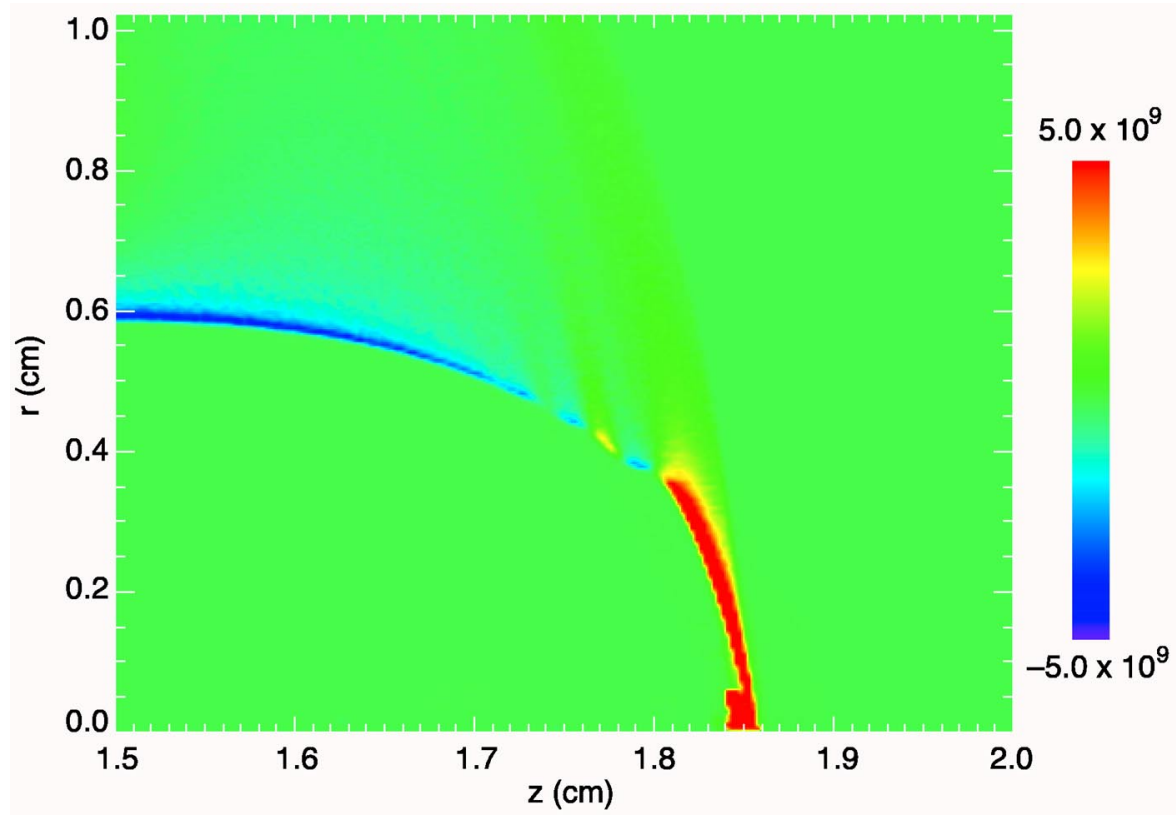

FIG. 2. (Color) Color intensity map of the plasma and beam longitudinal current density as a function of $r$ and $z$, with green indicating the absence of current, red indicating forward electron motion, and blue backward electron motion. Plasma and beam conditions are in Fig. 1. For these conditions $e n_{0} c=5 \times 10^{9} \mathrm{~A} \mathrm{~m}^{-2}$, the maximum amplitude in the color scale. A large forward current density is present in the beam region. 
density perturbations, is extremely challenging to study analytically. In order to extract some information from the analysis of this system, we therefore begin with some simplifying assumptions. First, we assume that the drive beam is traveling at an ultrarelativistic velocity $(v \simeq c)$, which implies by causality that no signals can propagate forward in the beam frame. Additionally, since we are primarily interested here in the effect of the plasma response on the beam itself, i.e., the collective energy loss of the beam, we only have to evaluate this solution for the induced fields over the extent of the beam. Coupled with our assumption of an infinitesimally short beam, it can be seen that the physical description of the system needs to be evaluated only on a plane that moves with the beam. As noted above, we assume cylindrical symmetry, so that the beam charge is contained in a disklike region.

Before proceeding with a formal discussion of our analysis, we begin with a qualitative discussion of its features. To begin, we note that blowout itself - the radial expulsion of plasma electrons from the beam regionwhich will be induced in the case of large $\tilde{Q}$, cannot play a role in how we generate our solution, because it occurs only after the passage of the $\delta$-function beam. Because of this, the only modification to the plasma density in the beam region that we will have to consider in the analysis is due to longitudinal plasma currents.

The most notable nonlinear feature of our solution is the fact that in the large beam charge case, the electrons develop a large forward momentum. This is due to their interaction with the transverse crossed electric and magnetic fields. It is in the opposite direction of the wellknown neutralization current that develops in the finitelength beam case, which arises in response to the induced $E_{z}$. The reason for this is that in our case, with our assumed beam distribution, we have the condition $E_{z} \ll$ $E_{r}, H_{\phi}$; in fact, in the region in direct contact with the beam, the longitudinal field is finite, while the transverse fields are singular in the ultrarelativistic, $\delta$-function beam limit.

We proceed to solve the problem of the cold-plasma fluid response to the passage of an ultrashort, highly relativistic electron beam, in dimensionless variables. This method allows us to clearly highlight the role of the dimensionless beam charge $\tilde{Q}$. As noted above, we expect linear behavior to result when $\tilde{Q} \ll 1$, while in cases that $\tilde{Q} \gg 1$ the system will be strongly nonlinear. In the dimensionless analysis, distance is measured in units of the plasma skin depth $k_{p}^{-1}$, fields are in units of the cold wave-breaking field $E_{\mathrm{WB}}=m_{e} c \omega_{p} / e$, velocities are in units of $c$, and momenta are in units of $m_{e} c$. The plasma density and currents are in units of $n_{0}$ and $e n_{0} c$, respectively. Use of dimensionless quantities is identified, as we have in the case of the beam charge above, by placing a tilde over the variable, such as $\tilde{n}=n / n_{0}$.
Using the standard assumption common to wakefield analyses of a static solution [17] in the beam frame, the solution has "wavelike" form, and is only a function of $\xi=k_{p}(z-c t)$, the longitudinal coordinate in the beam frame. With this assumption, we have $\partial / \partial z=-\partial / \partial c t$, and Maxwell's equations can be combined to yield

$$
\begin{gathered}
\frac{\partial^{2} \tilde{H}_{\phi}}{\partial \tilde{r}^{2}}+\frac{1}{\tilde{r}} \frac{\partial \tilde{H}_{\phi}}{\partial \tilde{r}}-\frac{\tilde{H}_{\phi}}{\tilde{r}^{2}}=-\frac{\partial \tilde{J}_{r}}{\partial \xi}+\frac{\partial \tilde{J}_{z}}{\partial \tilde{r}} \\
\frac{\partial \tilde{E}_{z}}{\partial \tilde{r}}=\tilde{J}_{r}
\end{gathered}
$$

and

$$
\frac{\partial}{\partial \xi}\left(\tilde{E}_{r}-\tilde{H}_{\phi}\right)=\tilde{J}_{r}
$$

The quantity $H_{\phi}$ will be central to our analysis, and we now introduce its integral over the beam region, assumed to be centered at $\xi=0$, as $\mathbf{H}=\int_{-\epsilon}^{+\epsilon} \tilde{H}_{\phi} d \xi$. We further anticipate a solution where $\tilde{H}_{\phi}$ and $\tilde{E}_{r}$ are singular over the extent of the beam (as they are in vacuum in the ultrarelativistic limit), while the response to that impulse yields plasma quantities $\tilde{J}_{r}, \tilde{J}_{z}$, and $\tilde{n}$ that remain finite, along with $\mathbf{H}$. Assuming a beam with surface charge that is uniformly distributed within a hard-edge radius $\tilde{a}$, Eq. (2) can now be integrated with respect to $\xi$ to give

$$
\frac{\partial^{2} \mathbf{H}}{\partial \tilde{r}^{2}}+\frac{1}{\tilde{r}} \frac{\partial \mathbf{H}}{\partial \tilde{r}}-\frac{\mathbf{H}}{\tilde{r}^{2}}-\left.\tilde{J}_{r}\right|_{\xi=-\epsilon}=\frac{\tilde{Q}}{\pi \tilde{a}^{2}} \delta(\tilde{r}-\tilde{a}),
$$

where the term involving $\tilde{J}_{z}$ has been dropped because it is the integral of a finite quantity over an infinitesimal $\xi$ interval.

We wish to evaluate the relativistically correct expression for $\tilde{J}_{r}$ in terms of the fields. Using the fact that the terms on the left-hand side of Eq. (4) are singular, while the right-hand side is finite, we can approximate $\tilde{E}_{r} \simeq \tilde{H}_{\phi}$ in the region of interest. We may then proceed to make use of the solution of electron motion in perpendicular, equal and constant $E$ and $H$ fields from Landau [19]. In using this solution, we model our longitudinal $\delta$ function as a constant flattop function contained within a region which is then allowed to approach the limit that its width tends to zero. In order for the Landau approach to be adopted, the fields experienced by the plasma electron must not vary in $r$ during the beam's passage. Thus we are assuming that the effect of a plasma electron's radial motion which allows it to sample the radial nonuniformity in the fields can be neglected, an assumption which will be verified ex post facto.

With these assumptions, and with the initial condition that the plasma electron is at rest before the beam's arrival, this solution yields

$$
\left.\tilde{p}_{r}\right|_{\xi=-\epsilon}=\mathbf{H},
$$


and

$$
\tilde{p}_{z}=\frac{\tilde{p}_{r}^{2}}{2}
$$

Equation (6) indicates that the total radial momentum kick is equal to the field integral $\mathbf{H}=\int_{-\epsilon}^{+\epsilon} \tilde{H}_{\phi} d \xi=$ $\int_{-\epsilon}^{+\epsilon} \tilde{E}_{r} d \xi$. The total longitudinal kick is quadratic in this quantity, for obvious reasons. As it arises from the magnetic force on the plasma electron, it must be proportional to the product of the radial velocity and the magnetic field, both of which are linear in $\mathbf{H}$.

In order to evaluate $\tilde{J}_{r}$, we first write the radial plasma electron velocity $\tilde{\boldsymbol{v}}_{r}$ using Eq. (6) as

$$
\left.\tilde{\boldsymbol{v}}_{r}\right|_{\xi=-\epsilon}=\frac{\mathbf{H}}{\sqrt{1+\mathbf{H}^{2}+\frac{1}{4} \mathbf{H}^{4}}}=\frac{\mathbf{H}}{1+\frac{1}{2} \mathbf{H}^{2}} .
$$

The plasma electron density is found with the help of the continuity equation, noting that the contribution from the radial motion can be neglected, as

$$
\tilde{n}=\left(1-\tilde{\boldsymbol{v}}_{z}\right)^{-1}=1+\frac{1}{2} \mathbf{H}^{2} .
$$

We then arrive at the following expression for the radial plasma current density immediately behind the beam:

$$
\tilde{J}_{r}=\tilde{n} \tilde{\boldsymbol{v}}_{r}=\left(1+\frac{1}{2} \mathbf{H}^{2}\right) \cdot \frac{\mathbf{H}}{1+\frac{1}{2} \mathbf{H}^{2}}=\mathbf{H} .
$$

It should be noted that a linear, nonrelativistic analysis of the plasma response in our case gives an expression identical to the final result in Eq. (10), as in such an analysis the electron density is unchanged $(\tilde{n}=1)$, while the radial velocity is simply proportional to the radial momentum, $\tilde{\boldsymbol{v}}_{r}=\tilde{p}_{r}=\mathbf{H}$.

With the simple result of Eq. (10) substituted into Eq. (5), we obtain a linear, inhomogeneous modified Bessel equation,

$$
\frac{\partial^{2} \mathbf{H}}{\partial \tilde{r}^{2}}+\frac{1}{\tilde{r}} \frac{\partial \mathbf{H}}{\partial \tilde{r}}-\frac{\mathbf{H}}{\tilde{r}^{2}}-\mathbf{H}=\frac{\tilde{Q}}{\pi \tilde{a}^{2}} \delta(\tilde{r}-\tilde{a}),
$$

which has the solution

$$
\mathbf{H}(\tilde{r})=\frac{\tilde{Q}}{\pi \tilde{a}} \begin{cases}K_{1}(\tilde{a}) I_{1}(\tilde{r}) & (\tilde{r}<\tilde{a}), \\ K_{1}(\tilde{r}) I_{1}(\tilde{a}) & (\tilde{r}>\tilde{a}),\end{cases}
$$

where $I_{1}$ and $K_{1}$ are modified Bessel functions. The most surprising part of this result is that Eq. (11), and thus its solution, Eq. (12), depends linearly on $\tilde{Q}$. There is no nonlinear dependence to the field excitation, despite the nonlinearity of the plasma electrons' dynamical response. The linearity in the plasma response, as measured by the field integral $\mathbf{H}$, is a consequence of the fact that the fully nonlinear transverse current response is linear in $\mathbf{H}$. The microscopic basis for this behavior is discussed further below.
We are ultimately interested not in the transverse magnetic field integral, but in $\tilde{E}_{z}$ directly behind the beam, which is found by integrating Eq. (3) over the radial coordinate, while also employing Eq. (12),

$$
\begin{aligned}
\left.\tilde{E}_{z}(\tilde{r})\right|_{\xi=-\epsilon} & =\int_{\infty}^{\tilde{r}} \tilde{J}\left(\tilde{r}^{\prime}\right) d \tilde{r}^{\prime}=\int_{\infty}^{\tilde{r}} \mathbf{H}\left(\tilde{r}^{\prime}\right) d \tilde{r}^{\prime} \\
& =\frac{\tilde{Q}}{\pi \tilde{a}^{2}} \begin{cases}1-\tilde{a} K_{1}(\tilde{a}) I_{0}(\tilde{r}) & (\tilde{r}<\tilde{a}), \\
\tilde{a} I_{1}(\tilde{a}) K_{0}(\tilde{r}) & (\tilde{r}>\tilde{a}) .\end{cases}
\end{aligned}
$$

In the limit that $\tilde{a} \ll 1$, the field inside of the disk region is nearly constant and given by

$$
\begin{aligned}
\left.\tilde{E}_{z}(\tilde{r})\right|_{\xi=-\epsilon} & \simeq \frac{\tilde{Q}}{\pi \tilde{a}^{2}}\left[1-\tilde{a} K_{1}(\tilde{a})\right] \\
& \simeq \frac{\tilde{Q}}{2 \pi}\left[\ln \left(\frac{2}{\tilde{a}}\right)-0.577 \cdots\right],
\end{aligned}
$$

which is to leading order proportional to $\tilde{Q} / 2 \pi$. In physical units we may write Eq. (14) as

$$
\left.e E_{z}\right|_{\xi=-\epsilon} \simeq 2 e^{2} k_{p}^{2} N_{b} \ln \left(\frac{1.123}{k_{p} a}\right) .
$$

Because Eq. (10) is identical to the form of $\tilde{J}_{r}$ obtained in the linear approximation, this expression is identical to that obtained in linear treatments [20]. Note also that because the analysis from the point of Eq. (12) forward through Eqs. (13) and (15) is identical to that of the linear PWFA, one may easily generalize the results to account for arbitrary radial beam profiles $[2,15,16]$, not just the uniform surface charge density considered here.

With our solution for the relevant physical quantities in hand, we now reexamine a critical approximation made earlier, that of neglecting the possible radial displacement of the plasma electron during the beam passage. The change in radius of a plasma particle during the passage of the beam is, taking the $\delta$-function limit,

$$
\Delta \tilde{r}=\int \frac{\tilde{v}_{r}}{1-\tilde{v}_{z}} d \xi \leq \lim _{\epsilon \rightarrow 0} \int_{-\epsilon}^{+\epsilon} \mathbf{H} d \xi=0 .
$$

The integral on the far right-hand side of Eq. (16) vanishes because the integrand is finite, while the integration interval is allowed to go to zero. Thus, we are justified in dropping the radial term in the continuity equation, as well as the effects of radial gradients in the fields, in the $\delta$-function limit. The validity of this approximation for finite-length beams is analyzed in Appendix B.

As a way of further investigating the response of the plasma to the beam charge, and to establish the selfconsistency of our results, we now examine the energy content of the excitation left in the beam's wake. The energy per unit length which must be supplied by the beam is found by integrating the energy density contained in both the plasma motion and field over the plane directly behind the beam: 


$$
\begin{aligned}
\frac{d \tilde{U}}{d \tilde{z}}= & 2 \pi \int_{0}^{\infty}\left[\sqrt{1+\tilde{p}_{r}^{2}+\tilde{p}_{z}^{2}}-1\right] \tilde{n}\left(1-\tilde{\boldsymbol{v}}_{z}\right) \tilde{r} d \tilde{r} \\
& +2 \pi \int_{0}^{\infty} \frac{1}{2} \tilde{E}_{z}^{2}(\tilde{r}) \tilde{r} d \tilde{r} \\
= & \pi\left[\int_{0}^{\infty} \mathbf{H}^{2}(\tilde{r}) \tilde{r} d \tilde{r}+\int_{0}^{\infty} \tilde{E}_{z}^{2}(\tilde{r}) \tilde{r} d \tilde{r}\right] .
\end{aligned}
$$

In arriving at this expression we have used the fact that just behind the beam $\tilde{H}_{\phi}=\tilde{E}_{r}=0$ and does not contribute to the energy density at that point. Also, the mechanical energy density $\tilde{n}(\gamma-1)$ overtaken by the beam per unit length must be multiplied by $\left(1-\tilde{v}_{z}\right)$. Evaluation of the integrals Eq. (17) yields, in the limit that $\tilde{a} \ll 1$,

$$
\begin{aligned}
\lim _{\tilde{a} \rightarrow 0} \frac{d \tilde{U}}{d \tilde{z}} & \simeq \frac{\tilde{Q}^{2}}{2 \pi \tilde{a}^{2}} \int_{\tilde{a}}^{\infty}\left[K_{0}^{2}(\tilde{r})+K_{1}^{2}(\tilde{r})\right] \tilde{r} d \tilde{r} \\
& =\left.\frac{\tilde{Q}^{2}}{2 \pi \tilde{a}^{2}}\left[1-\tilde{a} K_{1}(\tilde{a}) I_{0}(\tilde{a})\right] \simeq \frac{1}{2} \tilde{Q} \tilde{E}_{z}\right|_{r=0, \xi=-\epsilon}
\end{aligned}
$$

as expected.

We have derived a result in which the fields, and thus the energy loss rate of the beam, scale linearly with $\tilde{Q}$, while the plasma dynamics are very nonlinear for high values of $\tilde{Q}$. The nonlinearity is based in large density perturbations, as well as relativistic effects. To relate the degree of nonlinearity in the physical system to $\tilde{Q}$, we can use Eq. (12) to obtain the function $\mathbf{H}(r)$ for a given beam radius. This can be used to obtain $\gamma$ and $\tilde{n}$ as, $\gamma(\xi=-\epsilon)=1+\frac{1}{2} \mathbf{H}^{2}, \quad$ and $\quad \tilde{n}(\xi=-\epsilon)=1+\frac{1}{2} \mathbf{H}^{2}$, which can both be large compared with unity with a suitable choice of $\tilde{Q}$. This is in contrast with linearized plasma assumptions which require $\delta n / n \ll 1$ and $\vec{p} \simeq$ $m \vec{v}$ (and, therefore, $\gamma \simeq 1$ ).

The linear proportionality to $\mathbf{H}$ [and thus ultimately to $\tilde{Q}$ through Eqs. (12) and (13)] of the induced radial plasma current density indicated in Eq. (10) arises from two effects which cancel each other. The radial velocity $\tilde{\boldsymbol{v}}_{r}$ saturates at a value well below unity, yet the density enhancement due to longitudinal motion exactly makes up for this saturation, and the induced $\tilde{J}_{r}$ remains linear in $\tilde{Q}$. This forward motion is a consequence of the particle motion under large, crossed electric and magnetic fields: the initial radially outward motion of the plasma electrons is converted to longitudinal motion by the effects of the azimuthal magnetic field. This is similar to the scenario from laser wakefield acceleration (LWFA), where the electromagnetic pressure of a short, intense laser gives rise to a density enhancement in the laser's leading edge. A comparison of mathematical and physical aspects of the present scenario to a previous analysis of the case of the LWFA [21] is given in Appendix A.

It should be noted that, despite it being readily observable in simulation, the initial forward motion of plasma electrons due to an intense beam has not yet been discussed in the literature. In fact, it is a striking result of the nonlinear analysis of the infinitesimally short beam, while standard use of linear theory would indicate that the direction of longitudinal plasma electron motion is always counter to the beam motion. Even at small amplitudes $(\tilde{Q} \ll 1)$ where one would expect the linear results to hold, the present results indicate a small but finite initial forward push of the plasma electrons during the passage of a $\delta$-function beam. It is reasonable to inquire how this effect has been overlooked in previous, linear analyses.

From our discussion above, it is clear the forward push arises from a simple mechanism: plasma electrons in the beam region are pushed radially by the strong radial electric field and then deflected forward by the azimuthal magnetic field. The standard linearization of the plasma electron cold fluid equations [2,15], which is performed in the most basic treatments of the beam-plasma interaction, ignores the effect of the magnetic field inside of the beam region. This is only consistent with linearization if one assumes that the beam charge density is a small quantity $\left(n_{b} \ll n_{0}\right)$ and may thus be treated perturbatively. On the other hand, the linearized treatment has been employed even for infinitesimally short, finite charge beams [2], in which case the beam density is singular, and its magnetic field may not be treated as a perturbation. In short, the linear treatment of a $\delta$-function beam in plasma misses a term arising from the beam's magnetic field that must be present in a self-consistent first order analysis. Inclusion of such a term in the linear analysis produces an initial forward momentum impulse, just as has been found here in the fully nonlinear treatment. For small beam charge $(\tilde{Q} \ll 1)$, this effect may indeed be neglected, as the initial forward velocity of the plasma electron is quadratic in the small number $\tilde{Q}$, while the subsequent deceleration of the plasma electron is linear in $\tilde{Q}$.

\section{DISCUSSION AND CONCLUSIONS}

The blowout regime has been noted to have several anomalous characteristics, which the analysis above clarifies. One may first observe that, as seen in the simulations of Sec. II, strong forward motion of plasma electrons occurs in the region of intense, ultrashort beams in plasma. Further, this forward motion results in an increase in plasma electron density (and thus longitudinal current density) in the longitudinal region close to the beam, as also observed in simulation. An analytical basis for these effects has been given in Sec. III, which shows that the effect is due to the inclusion of magnetic forces in the nonlinear, fully relativistic analysis of the beamplasma interaction in the longitudinal $\delta$-function limit. We have in the process employed a single parameter, the normalized charge $\tilde{Q}$, which identifies when such a bunched beam may be expected to give rise to nonlinear motion in the plasma. The most curious attribute of these dynamics is that it allows the transverse current excited 
and thus the decelerating electron field experienced by the beam to be linearly proportional to $\tilde{Q}$, even for $\tilde{Q} \gg 1$. As a result, the decelerating field on a longitudinal $\delta$-function beam as calculated in the full nonlinear theory is the same as that derived by the simple linear treatment. The linearity of the excited $\tilde{J}_{r}$ is seen from our analysis to arise from the cancellation of the effects of two nonlinear phenomena, the limiting of the transverse velocity by relativistic effects, and the forward motion and density increase of the plasma electrons.

This brings us to a second curious characteristic of the blowout regime - the persistence of linear scaling in the excited wakefields as a function of $\tilde{Q}$, previously noted in the literature [6-9]. As the result obtained in the present work concerns only longitudinal $\delta$-function beams, the linear scaling of decelerating wakefields rigorously applies only in this limit. For actual experimental systems, however, the bunch has finite length, and a study of the scaling of wakefields must be performed with alternate methods. This has been done in a companion paper [5], which employs PIC simulations to explore the scaling of both decelerating and accelerating wakefields with charge $(\tilde{Q})$ in the nonlinear limit, for both ultrashort beams, and for beams with $k_{p} \sigma_{z} \approx 1$. It is found in these simulations that the effects of initial forward motion of the plasma electrons, along with the accompanying density increase, are present in both cases. In addition, it is observed that the beam energy loss predicted by simulations indeed scales nearly linearly with charge until $\tilde{Q}$ well in excess of unity, but at high enough $\tilde{Q}$ notable deviations from linear scaling of the induced longitudinal fields are present, and the wakefield amplitude tends to saturate.

Despite the need to utilize simulations to explore further aspects of the plasma wakefield excitation with finite-length $\left(k_{p} \sigma_{z} \approx 1\right)$ beams, some extensions to our understanding of the underlying physics at play may be deduced from the present analysis. In Appendix B, we examine the analytical aspects of weakly violating the assumption of infinitesimal beam length, to determine the degree to which we expect our results to hold for very short, but finite-length beams. In addition, we use a numerical solution of the fluid equations to illustrate the degradation of the deceleration as a function of beam length in a system where $\tilde{Q}$ exceeds unity. Beyond these mathematical exercises, it can be stated that the weakening of the fields in the case of finite bunch length is due to both the radial expulsion of the plasma electrons, and the longitudinal deceleration of the plasma electrons on the time scale of the beam passage. Both effects can be seen from the PIC simulations in Figs. 1 and 2, where the beam is quite short, but the beam charge is extremely large, $\tilde{Q}=200$. It can be seen that the blowout of the plasma electrons proceeds immediately behind the beam. The strong forward pushing of plasma electrons, which allows them to stay in contact with the beam charge for a longer time (proportional to $\left(1-\tilde{\boldsymbol{v}}_{z}\right)^{-1}$ ) is quickly reversed by the influence of the induced longitudinal electric field behind the beam.

The effects of radial blowout and deceleration within the bunch, and their degrading impact on the excited longitudinal fields, are clarified in Ref. [5]. This computational study extends and deepens the results given here, in the context of more experimentally relevant systems. In this regard, the studies of the plasma response as a function of $\tilde{Q}$ are most relevant - in order to produce higher wakefield amplitudes with constant available beam charge, the bunch is compressed to shorter $\sigma_{z}$, thus allowing use of larger $k_{p}$, and raising $\tilde{Q}$. As experiments at Stanford are now being considered which may achieve $\tilde{Q} \simeq 80$ or more, further studies of the plasma response for very high charge are indeed urgent.

\section{ACKNOWLEDGMENTS}

This work was supported by the U.S. Department of Energy Grant No. DE-FG03-92ER40693 and the U.S. Department of Education Grant No. G1A-62056.

\section{APPENDIX A: POTENTIAL-BASED SOLUTION}

Throughout this paper we have used the electromagnetic field quantities directly to solve for the plasma response. However, treatments of plasma wakes have alternatively based their analyses on the vector and scalar potentials, $A$ and $\Phi$. In this Appendix, we will tie our analysis to the vector potential-based models.

Many analyses make use of the Coulomb gauge, including those of Chen et al. [1] and Sprangle et al. [21]. In this gauge, the most common solution for the potential $\Phi$, which is governed by the Poisson equation, is given in terms of the charge density $\rho$ by use of the Green function formalism, as

$$
\Phi(x, t)=\int \frac{\rho\left(x^{\prime}\right)}{\left|x-x^{\prime}\right|} d^{3} x^{\prime} .
$$

This expression requires one to solve for $\rho$ everywhere before evaluating $\Phi$. In the case of Chen et al., this is made possible by performing a linearization of the plasma equations of motion and continuity equation, while in the analysis due to Sprangle et al., the simplification of having to solve the Poisson equation only in 1D allows $\rho$ to be expressed simply as a function of $A$ and $\Phi$. In more complex scenarios, such as the present $2 \mathrm{D}$ nonlinear case, this approach leads to complications in the analysis in that the equations for $A$ and $\Phi$ are coupled. Thus, following the lead of the numerical analysis of plasma wakefields given by Keinigs and Jones [14], we will perform our potential analysis in the Lorentz gauge.

Using ultrarelativistic beam assumptions in the Lorentz gauge, the vector potential is given by

$$
\nabla_{\perp}^{2} \vec{A}(r, \xi)=-\frac{4 \pi}{c} \vec{J}(r, \xi),
$$


where $\nabla_{\perp}^{2}$ is the transverse Laplacian. Substitution of $\nabla^{2} \vec{A}$ with $\nabla(\nabla \cdot \vec{A})-\nabla \times \nabla \times \vec{A}$, and then taking the curl of the resulting equation gives the result

$$
\frac{\partial}{\partial \tilde{r}} \frac{1}{\tilde{r}} \frac{\partial}{\partial \tilde{r}} \tilde{r} \tilde{H}_{\phi}=\frac{\partial}{\partial \tilde{r}} \tilde{J}_{z}+\frac{\partial}{\partial \xi} \tilde{J}_{r},
$$

which is equivalent to our earlier expression (2). Explicitly evaluating the components of the potential $(\tilde{A}$ and $\tilde{\Phi}$ ) equations summarized by Eq. (A2) gives, with normalized potentials indicated by the tildes,

$$
\begin{aligned}
& \frac{\partial}{\partial \tilde{r}} \frac{1}{\tilde{r}} \frac{\partial}{\partial \tilde{r}} \tilde{r} \tilde{A}_{r}(\tilde{r}, \xi)=\tilde{J}_{r}(\tilde{r}, \xi), \\
& \frac{1}{\tilde{r}} \frac{\partial}{\partial \tilde{r}} \tilde{r} \frac{\partial}{\partial \tilde{r}} \tilde{A}_{z}(\tilde{r}, \xi)=\tilde{J}_{z}(\tilde{r}, \xi), \\
& \frac{1}{\tilde{r}} \frac{\partial}{\partial \tilde{r}} \tilde{r} \frac{\partial}{\partial \tilde{r}} \tilde{\Phi}(\tilde{r}, \xi)=\tilde{\rho}(\tilde{r}, \xi) .
\end{aligned}
$$

Equations (A4)-(A6) are still coupled through the current density $\vec{J}$, which depends on both components of $\vec{A}$ and $\Phi$.

In order to solve for $\tilde{A}_{r}$ in the case of a $\delta$ function in the time beam we equate, following previous arguments on both linear and nonlinear plasma response, $\tilde{J}_{r}$ with $\mathbf{H}$. We combine the result in Eq. (12) with Eq. (A4), to obtain

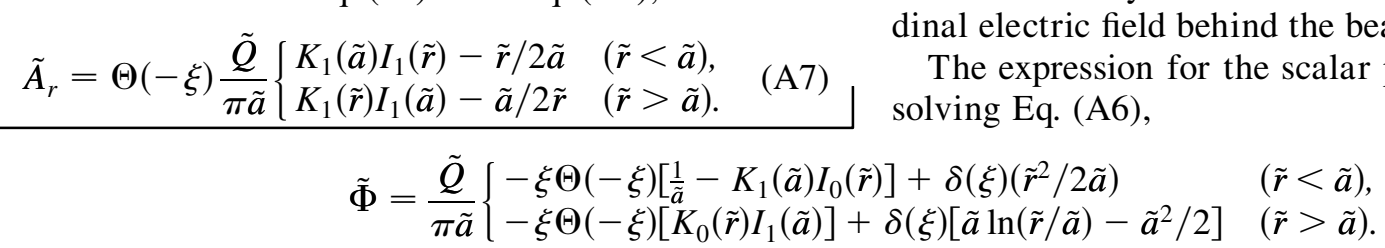

$$
\tilde{A}_{z}=\delta(-\xi) \frac{\tilde{Q}}{2 \pi \tilde{a}} \begin{cases}\tilde{r}^{2} / 2 \tilde{a} & (\tilde{r}<\tilde{a}) \\ \tilde{a} \ln (\tilde{r} / \tilde{a})-\tilde{a}^{2} / 2 & (\tilde{r}>\tilde{a})\end{cases}
$$

Note that the component $\tilde{A}_{z}$ is nonvanishing only at the longitudinal position of the beam itself. Further, it is straightforward to verify by taking the curl of the vector potential that one obtains the correct expression for the magnetic field inside of the beam [as indicated by the integrated value Eq. (12)], and that this magnetic field vanishes directly behind the beam. Note that the longitudinal electric field behind the beam due to $\tilde{A}_{z}$ vanishes.

The expression for the scalar potential is obtained by
The scalar potential inside of the beam mathematically yields a vacuumlike radial field [the $\delta$-function term in Eq. (A9)] that is canceled by an equal and opposite term in Eq. (A7); the only radial electric field left at the beam's longitudinal position is the self-consistent field of the beam in the plasma [whose longitudinal integral is given by Eq. (12)]. Note that the scalar potential directly behind the beam is zero, but it has a nonvanishing derivative. The longitudinal field directly behind the beam obtained from this derivative is identical to that given by Eq. (13).

The overall results obtained in this paper are similar in appearance to those that have been derived in the context of the one-dimensional (no variations allowed normal to propagation direction) laser-plasma interaction by Sprangle et al. [21]. Because of some confusion in formal discussions of the present results, we will remark on the similarities and differences between the two solutions. Indeed, there is an aspect of Sprangle's result dealing with single-particle motion that is general enough to be applicable to our case. Since we will be using this result in Appendix B to estimate effects due to a finite $E_{z}$, we will now explore this topic.

The treatment in Sprangle deals with the combination of a transverse wave described by the Cartesian coordi- nate fields $E_{x}(\xi)=H_{y}(\xi)$ and longitudinal charge separation which gives rise to $E_{z}$. We will use an asterisk to label the Coulomb gauge potentials in Cartesian coordinates $\left(A_{x}^{*}\right.$ and $\left.\Phi^{*}\right)$ to differentiate them from the Lorentz gauge axisymmetric quantities developed earlier. Thus, the transverse wave is represented by $A_{x}^{*}(\xi)$, and the charge separation is reflected in $\Phi^{*}(\xi)$, where neither of these quantities has any transverse variation. If we initially ignore the dynamics governing the evolution of $A_{x}^{*}$ and $\Phi^{*}$ (or equivalently the fields), and instead solve a problem in which the form of $H_{x}(\xi)$ and $E_{z}(\xi)$ is externally specified, then we may obtain a set of potentials that are consistent with these fields according to $\Phi^{*}=-\int E_{z}(\xi) d \xi$ and $A_{x}^{*}=-\int H_{y}(\xi) d \xi$. Following Sprangle's derivation, a particle evolving under the influence of such fields will have its motion described by

$$
\begin{gathered}
\gamma=\frac{1+\left(A_{x}^{*}\right)^{2}+\left(1+\Phi^{*}\right)^{2}}{2\left(1+\Phi^{*}\right)}, \\
\tilde{\boldsymbol{v}}_{z}=\frac{1+\left(A_{x}^{*}\right)^{2}-\left(1+\Phi^{*}\right)^{2}}{1+\left(A_{x}^{*}\right)^{2}+\left(1+\Phi^{*}\right)^{2}},
\end{gathered}
$$

with $\tilde{\boldsymbol{\beta}}_{x}=A_{x}^{*} / \gamma$. Additionally, in a 1D Cartesian system, 
one has as an immediate consequence of the continuity equation the relationship $\tilde{n}=\left(1-\tilde{\boldsymbol{v}}_{z}\right)^{-1}$, as in our Eq. (9).

Applying this approach to our problem in which the role of $E_{z}$ can be neglected (and thus $\Phi^{*} \simeq 0$ ), we obtain

$$
\tilde{\boldsymbol{v}}_{x} /\left(1-\tilde{\boldsymbol{v}}_{z}\right)=A_{x}^{*}
$$

which is equivalent to the result obtained earlier in Eq. (10) that specifies the linearity between $\tilde{J}_{r}$ and $\mathbf{H}$, a result that's central to our analysis. We comment that the equations developed by Sprangle, since they can be used in a very flexible manner by specifying an arbitrary $\xi$ dependence for both longitudinal and transverse fields, might be useful for solving a broader set of problems than the ones discussed here. The results above, which are obtained only considering the plasma electron dynamics in a specified crossed electric and magnetic field of equal magnitude, are analogous to ours. Consideration of how the plasma electron response enters into self-consistent determination of the fields illustrates the differences between our analysis and that of Sprangle et al.

We emphasize that the above results due to Sprangle et al., concerning the relationships between the plasma electron momenta and density, are analogous to ours only in a restricted scenario. They are related only when the charge separation in the plasma and therefore the scalar potential can be neglected, as is true in our analysis of the fields directly behind the $\delta$-function beam. Under these conditions, if one utilizes the conservation of transverse canonical momentum to make the substitution $\left(A_{x}^{*}\right)^{2}=\tilde{p}_{x}^{2}$ in Eqs. (A10) and (A11), then the results are equivalent to ours. The physical reason for this equivalence is that the roles of the transverse momenta, $\tilde{p}_{r}$ and $\tilde{p}_{x}$, are the same in the two treatments: they generate both $\tilde{p}_{z}$ and thus $\tilde{n}$.

We now discuss the differences between the Sprangle et al. treatment and the present analysis. Both analyses proceed to use the established relationships between the transverse momentum $\tilde{p}_{r}$ (or $\tilde{p}_{x}$ in the 1D Cartesian case), $\tilde{p}_{z}$, and $\tilde{n}$ to solve for the fields in their respective scenarios. In the present case of the cylindrically symmetric $\delta$-function beam, we must derive for the fields through knowledge of the simple relationship between $\mathbf{H}$ and $\widetilde{J}_{r}$, and the solution of the modified Bessel equation governing $\mathbf{H}$. The longitudinal electric field is then found by integration of Eq. (3). On the other hand, in the case of Sprangle et al., one must ultimately solve the 1D Poisson equation to obtain the longitudinal electric field.

In Sprangle et al. $E_{z}$ is formed entirely due to longitudinal charge separation (driven by $J_{z}$ ) as dictated by $\left(\partial^{2} / \partial \xi^{2}\right) \Phi^{*}=-4 \pi \rho$. The transverse current $J_{\perp}$ does not strongly couple as a source term to the field evolution equations. For short driver pulses, in fact, the role of $J_{\perp}$ can be neglected. Over longer time scales (many periods of the "fast" optical time scale) $J_{\perp}$ eventually makes a cumulative change in the evolution in $A_{x}^{*}$ and $\Phi^{*}$, which serves to evolve the wakefield $E_{z}$ on the slow time scale.
For our problem it is the quantity $J_{z}$ which can be ignored. Although longitudinal charge separation eventually does play a role farther back in the plasma, it is of negligible importance for computing the fields directly behind an infinitely short beam. The role of $J_{r}$, however, is quite important in our case in that it couples directly to the transverse fields. Unlike the 1D laser-driven case, this coupling manifests itself immediately, even when the beam is infinitely short. One consequence of this coupling is the fact that the plasma is shielded from the bare drive beam fields on a length scale of $k_{p}^{-1}$ in radius. The correct treatment of this phenomenon requires the solution of a differential equation in $r$ of the form of Eq. (5). Also, $J_{r}$ causes an immediate change in $E_{z}$ according to Eq. (3). Therefore, in our 2D cylindrically symmetric case, the method of longitudinal wakefield creation is through the coupling of the transverse current to an electromagnetic transient involving the transverse fields which in turn establish the longitudinal field.

The change in emphasis from $J_{z}$ to $J_{r}$ when going from the 1D Cartesian picture to the $2 \mathrm{D}$ axisymmetric case is rooted in the fact that in $1 \mathrm{D}$, the $\nabla \times \vec{H}$ term in the Maxwell's equations has a zero longitudinal component, and therefore this equation implies $\widetilde{J}_{z}=(\partial / \partial \xi) E_{z}$, which is equivalent to $(\partial / \partial \xi) \tilde{E}_{z}=\tilde{\rho}$. In the $2 \mathrm{D}$ picture, this component of $(\vec{\nabla} \times \vec{H})_{\phi}$ is no longer zero. This term is evaluated by combining both Maxwell equations to yield the result in Eq. (3), $(\partial / \partial \tilde{r}) \tilde{E}_{z}=\tilde{J}_{r}$. This difference between the two pictures influences many aspects of the calculation. For instance, an infinitely wide (1D) electron beam will have no transverse magnetic field, while a beam which is merely very wide may have an extremely large $H_{\phi}$.

We conclude by saying that, although there is a similarity in single-particle dynamics in our analysis and that of Sprangle et al., the global evolution of the fields, as well as much of the physics behind that evolution, is quite different.

\section{APPENDIX B: BUNCH LENGTH EFFECTS IN THE FLUID MODEL}

In this paper we have considered only the limit of a beam described by a temporal $\delta$ function. In order to apply our results to systems where the beam is very short (but finite) in time, we will now examine how quickly such a solution converges to the $\delta$-function case in the limit of small bunch length. Our approach will be perturbative in nature in that we will assume some part of our original solution remains intact while we estimate the conditions under which the extra terms arising from finite bunch effects can be neglected. In all cases, we assume that the beam is at least short enough to satisfy

$$
2 \epsilon \ll 1,
$$

which limits the effect of plasma oscillatory motion on 
the beam energy loss. The conditions developed below should be treated as additional constraints in the case of a high-charge beam.

We first treat single-particle motion in equal transverse fields $\left(E_{r}=H_{\phi}\right)$, but with non-negligible $E_{z}$ and finite radial motion. In order to simplify some of the integrals, the functional dependence of $E_{r}(r, \xi)$ is assumed to be the product of the radial dependence implied by Eq. (12), and a $\xi$ dependence that is constant in the interval from $\xi=$ $-\epsilon$ to $\xi=\epsilon$. As a plasma particle is pushed outward in radius, it will sample a relative decrease in the applied fields. We will solve for the particle motion in this case using the method outlined in Appendix A. Since that method does not allow for a transverse variation in field quantities, but does allow for an arbitrary longitudinal profile, we will "absorb" the transverse variations into a suitably modified longitudinal profile, which we term the effective fields. This is done under the constraint that the particles experience the same transverse and longitudinal fields as a function of time in both representations. The particle radius will evolve according to $\tilde{r}(\xi)=\tilde{r}_{0}+$ $\int\left(\beta_{r} / 1-\beta_{z}\right) d \xi$. We define the effective fields according to

$$
E_{r, \mathrm{eff}}(\xi)=E_{r}[r(\xi), \xi],
$$

and likewise for $H_{\phi}$ and $E_{z}$. We are most interested in solving for the transverse current, which, ignoring transverse convection terms in the continuity equation, is given in the notation of Appendix A by $J_{\perp}=A_{\perp}^{*} /(1+$ $\left.\Phi^{*}\right)$. In terms of our original fields, the radial current is given by

$$
\tilde{J}_{r}=\frac{\int_{-\epsilon}^{\epsilon} \tilde{H}_{\phi}[\tilde{r}(\xi), \xi] d \xi}{1+\int_{-\epsilon}^{\epsilon} \tilde{E}_{z}[\tilde{r}(\xi), \xi] d \xi},
$$

where the particle radius must be self-consistently computed according to $\tilde{r}(\xi)=\tilde{r}_{0}+\int_{0}^{\epsilon} \tilde{J}_{r}(\xi) d \xi$. If we require this radius to not change very much from its original value, we arrive at the condition,

$$
2 \epsilon \ll \frac{r}{\mathbf{H}},
$$

where factors of order unity have been dropped. Similarly, the effect of a finite $E_{z}$ will be minimized when the denominator of Eq. (B2) is kept at a value of unity, which yields the expression

$$
2 \epsilon \ll \frac{1}{\mathbf{H}},
$$

again dropping factors of order unity. The effect of $E_{z}$ is to provide a force in the direction opposite the beam motion (the neutralization current), which acts to diminish the density increase caused by the forward motion.

We now turn to the change in electron density due to radial convection. Including radial motion, the continuity equation reads

$$
\frac{\partial}{\partial \xi} \tilde{n}\left(1-\tilde{\boldsymbol{v}}_{z}\right)+\frac{1}{\tilde{r}} \frac{\partial}{\partial \tilde{r}}\left(\tilde{r} \tilde{J}_{r}\right)=0
$$

where in the absence of this motion $\tilde{n}\left(1-\tilde{\boldsymbol{v}}_{z}\right)=1$. After integrating over $\xi$, the contribution from the second term is seen to be negligible when

$$
2 \epsilon \ll\left(\frac{1}{\tilde{r}} \frac{\partial}{\partial \tilde{r}} \tilde{r} \mathbf{H}\right)^{-1} .
$$

This condition is not more restrictive than the previous one in the region outside the beam radius. However, inside the beam radius the functional form of $J_{r}$ is that of a uniform expansion accompanied by a lowering of the plasma electron density. This effect can be very strong in the case of a narrow beam. For example, when $\tilde{a}=$ 0.01 and $\tilde{Q}=100$, the right-hand side of the above expression has a value of $6 \times 10^{-4}$, where the minimum value of $1 / \mathbf{H}$ [for the purpose of evaluating Eq. (B3)] is 0.12 . If this condition is strongly violated, the plasma electrons in the beam volume will be blown out, resulting in a loss of energy coupling from beam to plasma.

We would also like to examine the impact on singleparticle motion when $E_{r} \neq H_{\phi}$. The difference between these two quantities is given by combining Eqs. (4) and (6), with the result that at the back of the bunch $\tilde{E}_{z}-$ $\left.\tilde{H}_{\phi}\right|_{\xi=-\epsilon}=2 \epsilon \mathbf{H}$. We further simplify the problem by assuming that the ratio between the field quantities $R=$ $E_{r} / H_{\phi}$ is a constant and is given by the field imbalance at the back of the bunch, given by $1-R=4 \epsilon^{2}$. The plasma electron motion can then be solved by the method of transforming to a frame where $E_{r}=0$, with a transformation velocity of $\beta_{t}=R$. Although the radial kick $\Delta p_{r}$ stays approximately unchanged, the longitudinal momentum after transforming back will be given by

$$
p_{z}=\beta_{t} \gamma_{t}^{2}\left(\sqrt{1-\frac{p_{r}^{2}}{\left(\beta_{t} \gamma_{t}\right)^{2}}}-1\right),
$$

where $\gamma_{t}=1 / \sqrt{1-\beta_{t}^{2}}$. This expression converges to the result given in Eq. (7) when $p_{r} \ll \beta_{t} \gamma_{t}$ and $\beta_{t} \simeq 1$. Using the fact that at the back of the bunch, $p_{r}=\mathbf{H}$ and $1 /\left(2 \gamma_{t}^{2}\right) \simeq 4 \epsilon^{2}$, the above condition can be stated as

$$
2 \epsilon \ll 1 / \mathbf{H} \text {. }
$$

In short, we can extend our infinitely short beam results to finite beams where the bunch length $2 \epsilon$ has a maximum size given by the above inequality conditions. For some of the effects we have examined, it is necessary to simply meet the above condition [Eq. (B8)]. However, additional constraints come into play at small radii and also for narrow beams, in the form of Eqs. (B3) and (B6). It should also be pointed out that, since effects arising from finite radial motion and finite $E_{z}$ all begin to be important for similar values of $\epsilon$, it would be difficult to develop an analytical theory that accounts for all effects 


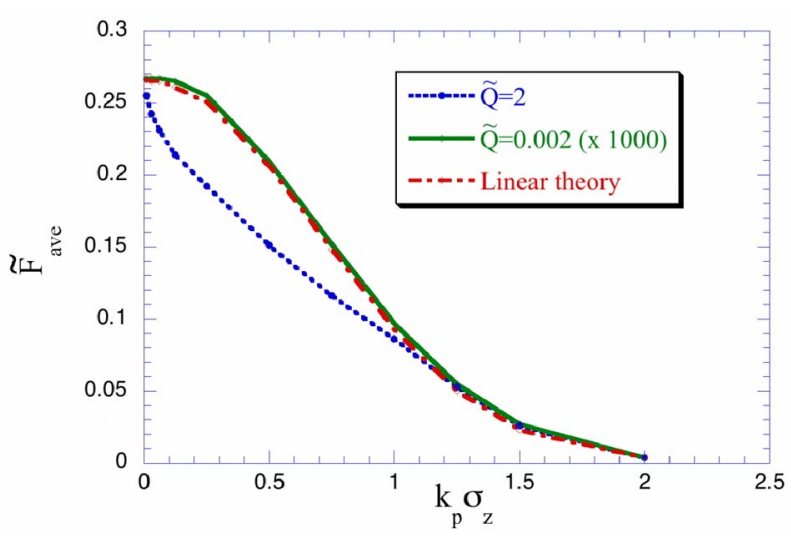

FIG. 3. (Color) The average normalized energy loss rate $\tilde{F}_{\text {dec }}=$ $e \bar{E}_{z} / m_{e} c \omega_{p}$ of an electron beam with $k_{p} a=0.2$, as a function of $k_{p} \sigma_{z}$, for $\tilde{Q}=0.002$ (diamonds, solid line) and $\tilde{Q}=2$ (circles, dotted line) from cylindrically symmetric fluid simulation and linear theory (diamonds, dashed line).

self-consistently; our approach based on perturbative analysis is, therefore, justified.

As an addition to our perturbative examination of the validity of the infinitesimal beam analysis, we have also examined finite beam length effects with numerical integrations of the two-dimensional fluid equations using a beam having a longitudinal charge distribution, $\rho_{b} \sim$ $\exp \left(-z^{2} / 2 \sigma_{z}^{2}\right)$, and exploring the limit that $k_{p} \sigma_{z} \rightarrow 0$. In order to connect with the $\delta$-function beam limit, and to accurately quantify the energy imparted to the plasma, we compare the average on-axis beam energy loss rate, $\left.\left(2 \pi \sigma_{z}\right)^{-1} \int e c E_{z}(\xi)\right|_{r=0} \exp \left(-c^{2} \xi^{2} / 2 \sigma_{z}^{2}\right) d \xi$ for these cases with linear theory. The predictions of linear theory are obtained from using Eq. (14) to give the Green function ( $\delta$-function response), and performing a convolution integral [2] over the Gaussian pulse, to give an average energy loss rate of $\left(\tilde{Q} / 2 \pi \tilde{a}^{2}\right)[1-$ $\left.\tilde{a} K_{1}(\tilde{a})\right] \exp \left(-k_{p}^{2} \sigma_{z}^{2}\right)$. The results of these simulations are shown in Fig. 3, which displays the average energy loss of a beam in the linear regime $(\tilde{Q}=0.002)$, a comparison to linear analytical theory, and the nonlinear regime $(\tilde{Q}=2)$. In the $\tilde{Q}=0.002$ case, the fluid simulations agree extremely well with analytical predictions. For the case with $\tilde{Q}=2$, the simulations disagree with linear theory over a broad range of pulse lengths, but converge to the linear theory in the short beam limit, as expected from the conclusions we have drawn from Eqs. (6) -(10) and (13).

Note that the numerical integration of the fluid equations is not easily stabilized when $\tilde{Q}>2$, and thus to perform further numerical investigations another tool must be adopted. The investigations using such a tool, electromagnetic PIC simulation, are discussed in the companion work to this paper [5]. We note their concep- tual importance is found in that the results of fluid integrations rely on a model that is simply connected to our analytical results (they are only a check on the validity of the derivation), while the PIC simulations are an entirely different, more complete model of the beam-plasma interaction. The PIC codes thus give a check on the physics of our analysis results, not solely the mathematics.

[1] Pisin Chen, J. M. Dawson, Robert Huff, and T. Katsouleas, Phys. Rev. Lett. 54, 693 (1985); 55, 1537 (1985).

[2] R. Ruth, R. D. Ruth, and A.W. Chao, in Laser Acceleration of Particles, edited by P. Channell, AIP Conf. Proc. No. 91 (AIP, New York, 1982).

[3] J. B. Rosenzweig, Phys. Rev. Lett. 58, 555 (1987).

[4] J. B. Rosenzweig, B. Breizman, T. Katsouleas, and J. J. Su, Phys. Rev. A 44, R6189 (1991).

[5] J. B. Rosenzweig, M. C. Thompson, R. Yoder, and N. Barov, in The Physics and Applications of High Brightness Electron Beams, edited by J. Rosenzweig, G. Travish, and L. Serafini (World Scientific, Singapore, 2003); Phys. Rev. ST Accel. Beams (to be published).

[6] J. B. Rosenzweig, in Proceedings of the 1992 Linear Accelerator Conference, Chalk River (Report No. AECL-10728, 1993).

[7] J. B. Rosenzweig et al., Nucl. Instrum. Methods Phys. Res., Sect. A 410, 532 (1998).

[8] N. Barov, J. B. Rosenzweig, M. E. Conde, W. Gai, and J. G. Power, Phys. Rev. ST Accel. Beams 3, 011301 (2000).

[9] S. Lee, T. Katsouleas, R. Hemker, and W. Mori, Phys. Rev. E 61, 7014 (2000).

[10] N. Barov et al., in Proceedings of the 2001 Particle Accelerator Conference, edited by P. Lucas and S. Webber (IEEE, Piscataway, NJ, 2002), p. 126.

[11] M. Hogan et al., Phys. Plasmas 7, 2241 (2000).

[12] S. Lee et al., Phys. Rev. ST Accel. Beams 5, 011001 (2002).

[13] D. L. Bruhwiler et al., Phys. Rev. ST Accel. Beams 4, 101302 (2001).

[14] Rhon Keinigs and Michael E. Jones, Phys. Fluids 30, 252 (1987).

[15] T. Katsouleas, Phys. Rev. A 33, 2056 (1986).

[16] P. Chen, Part. Accel. 20, 171 (1987).

[17] This implies that any transients from, e.g., the beam traversing a plasma boundary are no longer relevant, which means in practice that the beam is at least several skin depths $k_{p}^{-1}$ from the boundary [18].

[18] S. Mtingwa, Phys. Rev. A 37, 1668 (1988).

[19] L. D. Landau and E. M. Lifschitz, The Classical Theory of Fields (Pergamon Press, New York, 1975), 4th ed., p. 58.

[20] J. D. Jackson, Classical Electrodynamics (Wiley, New York, 1975), 2nd ed.

[21] P. Sprangle, E. Esarey, and A. Ting, Phys. Rev. A 41, 4463 (1990). 